\title{
Collagen Based Sponges for Wound Healing
}

\author{
M.Vallimeenal, L. Pravina, M. Anisha, N. Vigneshwari, M. Sushmitha, C. Jim Elliot
}

\begin{abstract}
Nowadays, wound healing is the common and simple problems occur in our society. Wound healing is the multi factorial process which includes inflammation, reepithelialisation, collagen deposition, and angiogenesis. Wound repair system is indispensable to enhance the proper functioning of skin. Normally, wound dressing is either momentary or lasting, wound dressings has been intended to improve the wound repair. Those wound dressing are required to wrap the large surfaced open wounds such as widespread burns, pressure ulcers, foot sores etc., wound healing substance should have some important features such as, high healing efficiency, anti-scar formation, providing favourable atmosphere for wound management. The collagen plays an important role in tissue formation and has more effect on wound healing. In this work collagen sponge is prepared from fish scales and to increase the antibacterial efficacy need extract has been added.
\end{abstract}

Keywords : Wound Healing, Multi factorial process, Wound dressing, Collagen.

\section{INTRODUCTION}

In the emerging tissue engineering field, the forthcoming development is completely based on biological materials to repair, maintain and protect the damaged tissues, because biological materials promotes the healing process by means of tissue regeneration. Hence these biological implants have become an important part in the biomedical research. These biological implants are used for variety of applications such as skin, bones, cartilages, blood vessels, heart tissues, intestines, tendons, ligaments and skeletal muscles. Based upon the applications required, the implants have to be made with their specific structural and mechanical properties.

Any detrimental or any physical injury to the skin may be termed as wound. The current growth in wound research management develops the awareness on wound repairing mechanism. Collagen is one among the best clinically efficient substances which have been utilized for wound repair process and skin regeneration process. Collagen has more effects on wound healing and blood coagulation.

Revised Manuscript Received on December 16, 2019.

* Correspondence Author

M. Vallimeenal, Department of Biomedical Engineering,, Kalasalingam Academy of Research and Education, Tamil Nadu, India.

L. Pravina, Department of Biomedical Engineering,, Kalasalingam Academy of Research and Education, Tamil Nadu, India.

Anisha.M*, Department of Biomedical Engineering, Kalasalingam Academy of Research and Education, Tamil Nadu, India. Email: anisha@klu.ac.in

N.Vigneshwari, Department of Biomedical Engineering, Kalasalingam Academy of Research and Education, Tamil Nadu, India. Email: vigneshwari.n@klu.ac.in

M.Sushmitha, Department of Biomedical Engineering,, Kalasalingam Academy of Research and Education, Tamil Nadu, India. sushmitha.m@klu.ac.in

C.Jim Elliot, Department of Biomedical Engineering, Kalasalingam Academy of Research and Education, Tamil Nadu, India.

Collagen has received wide-range of notice in recent years. Collagen has been utilized for plenty of clinical applications such as ossification, periodontal regeneration, vascular repair, skin repair, drug release etc. collagen's another conventional application is haemostasis.

Collagen, elastin and fibrous protein composed of extracellular matrix; they are available enormously in the skin, bones, cartilages, teeth muscles etc. The collagen was consumed as food enhances the function of immune system; it enhances the regeneration action of cell and makes strong joints. Collagens are eminent to sustain the metabolism activation and moisturization of skin. Collagen has excellent biodegradability.

Collagen has been processed into variety of shapes such as sheets, tubes, gel, sponges etc. the fibrillar structure is considered as a vital factor in the design of biomaterials. As they have the porous structure, they allow the tissue growth into the material. These collagen sponges are exclusively used for cell migration. Collagens also have some special features that they are adhesive to wet wounds. It absorbs huge quantity of tissue exudates. It prevents the moist environment. It also promotes the development of new granulation tissues and epithelium on the tissue injury. Collagens also have greater flexibility, durability, gas permeability, high tensile strength, low extensibility, fibre orientation. Collagen is commonly used for the wounds like extensive burns, foot ulcers, and pressure sore etc.

\section{RELATED WORKS}

Many researchers have aimed to achieve the greater extent in wound repair mechanism with collagen alone or with some other combinations in various applications. Thangavelu Muthukumar et al. (2014) prepared the collagen sponge by extracting the collagen from the fish scales and incorporated the macrotyloma uniflorum plant extract into the collagen sponge, then evaluated the physiochemical properties and characterization. This incorporation of plant extract increases the anti bacterial efficacy.

M.B.Fauzi et al. (2016) proposed few analyses for the collagen. Author extracted the collagen from the ovine tendon and purified it. The analysis for protein identification was performed via mass spectrometry; the chemical characterization was evaluated by Fourier transform infrared spectroscopy, x-ray diffraction and energy dispersive $\mathrm{x}$-ray spectroscopy. This analysis proves the existence of triple helix configuration of collagen. The FITR peaks at $1632 \mathrm{~cm}-1$ Karri et al.(2016) proposed the method for preparing the novel nanohybrid scaffolds. After the novel nanohybrid scaffolds are prepared and incorporated the curcumin in chitosan nanoparticles. CUR-CSNPs were estimated for partiucle size, zeta potential, SEM, differential scanning colorimeter, X ray. 


\section{Collagen Based Sponges for Wound Healing}

SEM is used to study the morphological characteristics. Mahmoud et al. (2016) prepared the norfloxacin loaded collagen/chitosan scaffolds were prepared and they were analyzed in terms of morphology, water absorption and retention capacity, biodegradation behavior. The morphology was viewed by SEM analysis which revealed that highly interconnected porous structure.

Sun et al. (2017) extracted the collagen from the pacific cod skin. These have good blood biocompatibility. They extracted two types of collagen from the pacific cod skin: Acid Soluble Collagen (ASC) and Pepsin Soluble Collagen (PSC). The SEM analysis and hemolysis assay in vitro indicated that these collagen sponges have great porosity and compatibility. They determine intrinsic velocity of ASC and PSC.

Lin et al. (2017) prepared the porous collagen discs and electro spinning the nanofibres into the collagen disc. These forms the bi-layered aligned composite. They observe the morphology of the nanofibres and pores by SEM analysis. Dollian et al.(1986) prepared the collagen sponges by characterizing the collagen with sodium dodecyl sulphate polyacrylamide gel and undergo SEM analysis to observe the factors that influences the pore size and configuration. Then mean and the standard deviation were calculated. The mean and the standard deviations were varies according to the variation in the freezing temperature.

Sun et al. (2018) done a comparison between the GTA treated collagen and DHT treated collagen. They extracted the collagen from the Nile tilapia skin and the effects of DHT and GTA cross linking on mechanical, biological properties and biodegradation behaviour were studied. They also evaluated the hygroscopicity assay and degree of cross linking.

Jinno et al. (2016) made a comparison between the collagen sponge and the collagen gelatine sponges. They prepared the collagen sponge and the collagen gelatine sponges as per the previous studies. The silicone solutions were used to make the silicone sheet which is used to cover the collagen gelatine sponge. The comparision denotes that collagen gelatine sponge can equal the efficacy of the conventional collagen sponge with bFGF.

Yan et al.(2018) prepared the collagen coated silk sponges and investigated that the regeneration of meniscus and analysing the collagen coated silk sponges characterization, invitro cyto compatibility by SEM analysis, microcomputed tomography, masson staining, fractional coefficient and mechanical testing. Then isolate the cells and allowed to culture, then they implanted the scaffolds into the rat and observe the meniscal regeneration.

Aravinthan et al. (2018) analysed that the collagen sponge hastens the wound healing process. They prepared the collagen sponge from the bovine Achilles tendon. Then they performed the western blot analysis which shows that the expression level of the vital growth factors like VEGF and TGF- $\beta$.these growth factors are mainly responsible for faster healing.

Mashiko et al. (2018) evaluated that rhCP as biomedical carrier for cellular regeneration therapy. They isolated the Stromal Vascular Fraction cells (SVF) and allowed to grown these cells in Dulbeccos modified eagle medium. These cells undergo trypsination. rhCp scaffolds are prepared and the cells are allowed to adhere and they are cultured. They are observed by SEM analysis. By means of analysing the histology of the cells, therapeutic effects are visualised.

Shazeeb et al. (2019) developing the MRI parameter. They fabricated the collagen sponge and implanted it and evaluated the tissue response. The implants are visualized by MRI parameters. The histological data are correlated with the MRI data. They help to interrupt the future, they helps to regenerate the damaged tissues and avoid unnecessary surgeries. Furthermore detailed survey has been made in [16] regarding collagen sponge preparation

\section{METHODOLOGY}

Block diagram of the proposed sponge preparation is depicted in Figure 1. Steps involved in the collagen sponge preparation are given as follows.

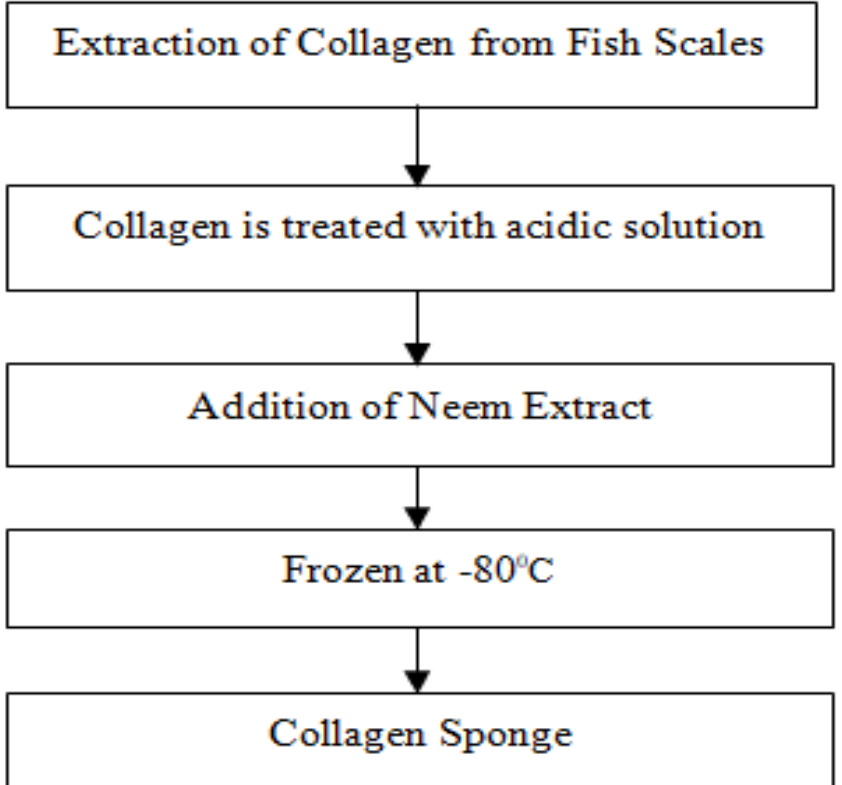

Fig. 1. Block Diagram of the Proposed Work

\section{A. Step 1: Extrication of Collagen from fish scales}

The collagen was extracted from the fish scales by the methods given further. Collected fish scales are shown in Figure 2. Initially, the fish scales are washed systematically with the distilled water, the added with the solvent substance consisting of $1 \mathrm{M} \mathrm{Nacl}, 0.05 \mathrm{M}$ Tris Hcl, 20.0mM EDTA with the $\mathrm{pH}$ of 7.5 to eliminate the unnecessary proteins and kept undisturbed for 48 hours. 


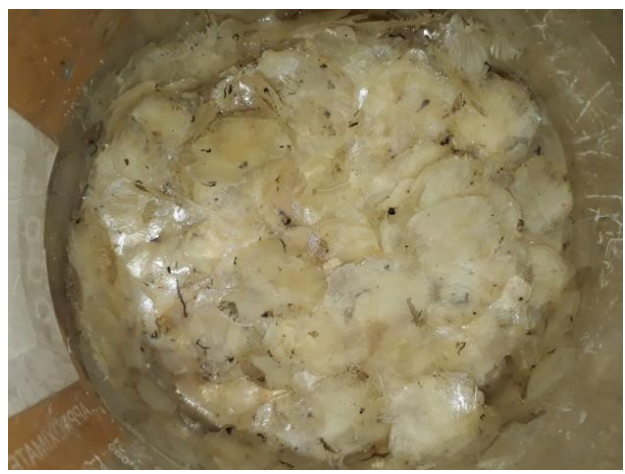

Fig. 2. Collected Fish Scales

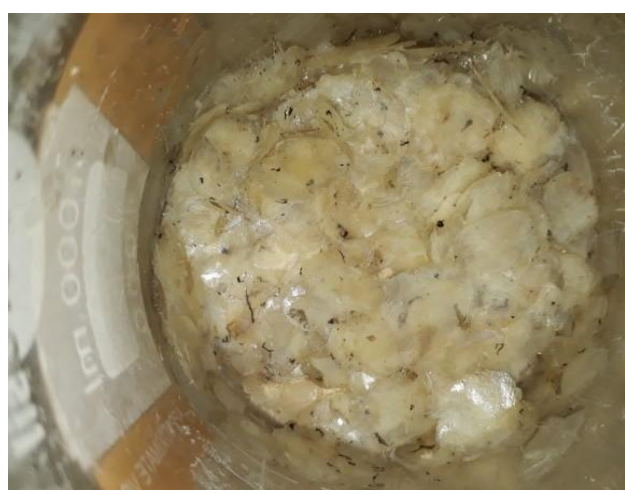

Fig. 3. Demineralised Fish Scales

After surveillance, remove the solvent substance and washed with the distilled water, then swap $0.05 \mathrm{M}$ EDTA to demineralise the fish scales and kept untouched for 48 hours, shown in Figure 3. After observation, the demineralised fish scales were treated with the $0.5 \mathrm{M}$ acetic acid and kept undisturbed for 48 hours, shown in Figure 4.

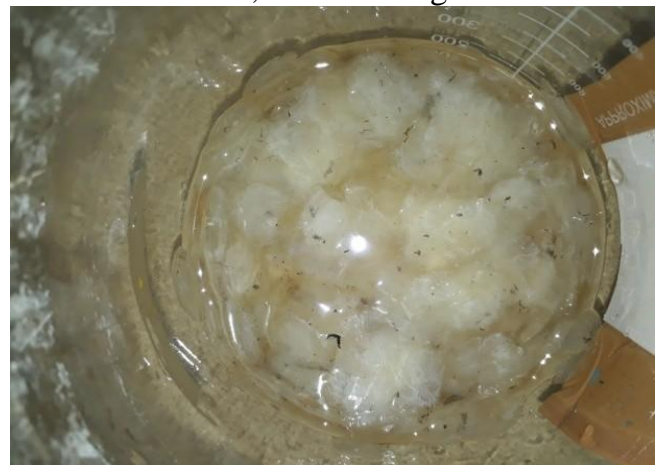

Fig. 4. Treated with Acidic Solution

The insoluble parts are filtered out. $\mathrm{NaCl}$ is added further to the filtrate to the ultimate concentration of $0.9 \mathrm{M}$ and kept uninterrupted for 24 hours. The suspensions are centrifuged at $8000 \mathrm{rpm}$ for 1 hour and resolubilized with $0.5 \mathrm{M}$ acetic acid and they are subjected to centrifugation. This process repeated for better result. Then the final solution is dialyzed by means of the dialysis membrane against $0.1 \mathrm{M}$ acetic acid and the distilled water for 24 hours. Finally, the solution is subjected to freeze dryer to acquire the final product as collagen.

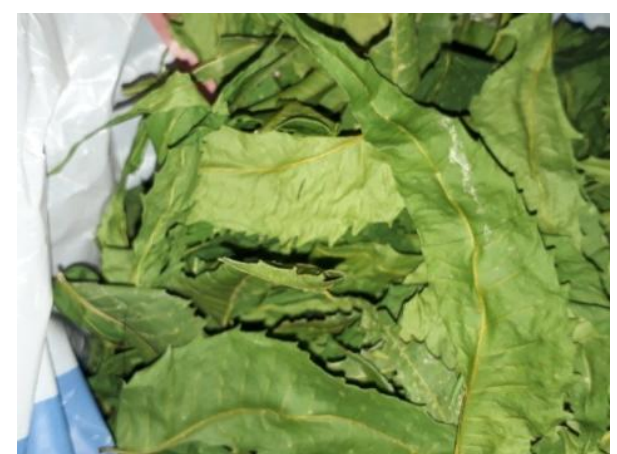

Fig. 5. Shade Dried Neem Leaves

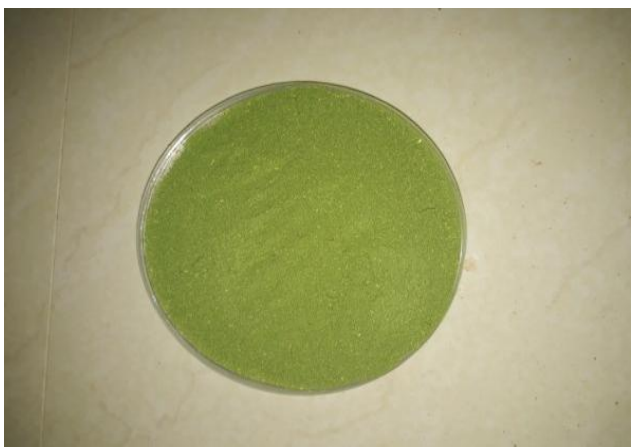

Fig. 6. Powdered Neem Leaves

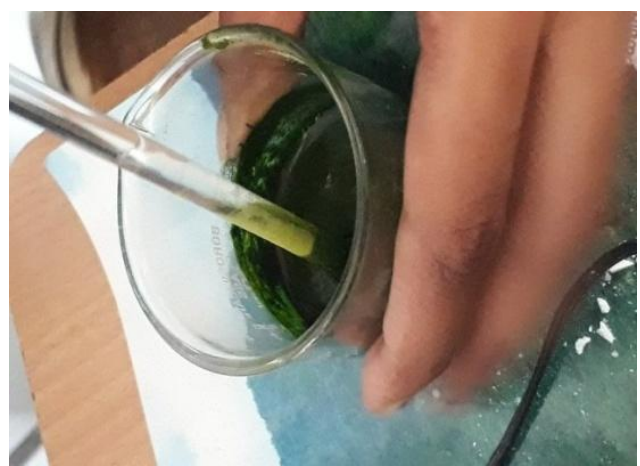

Fig. 7. Mixed with Methanol

\section{B. Step 2: Extrication of Neem Extract}

Fresh and healthy leaves of the Neem plants were gathered and cleansed from extraneous substances, shade dried shown in Figure 5, powdered (Figure 6) mechanically and hoard in air tight clean container. About $20 \mathrm{~g}$ of dusted substance was stopped in $40 \mathrm{ml}$ methanol for 72 hours in beaker and combination was stirred every 18 hour using a sterile glass rod (Figure 7). Filtrate was acquired 3 times with the aid of What man filter paper no 1 and the solvent was isolated by rotary evaporator (Figure 8 ) under reduced pressure at $<45^{\circ} \mathrm{C}$ temperature leaving a dark brown residue which was stored in air tight container at $40^{\circ} \mathrm{C}$ until utilize.

\section{Preparation/formation of Collagen Sponge}

Collagen sponges were prepared from collagen extracted from fish scales. The collagen was prepared in $0.5 \mathrm{M}$ acetic acid and left overnight at $4^{\circ} \mathrm{C}$. Frozen at $-80^{\circ} \mathrm{C}$ in aluminum weighing pans $(70-\mathrm{mm}$ diameter), and then lyophilized overnight. Then the collagen composed of spongy structure was obtained. Then the prepared nee $m$ extract were added to the collagen sponge and the 


\section{Collagen Based Sponges for Wound Healing}

frozen and lyo philized overnight.

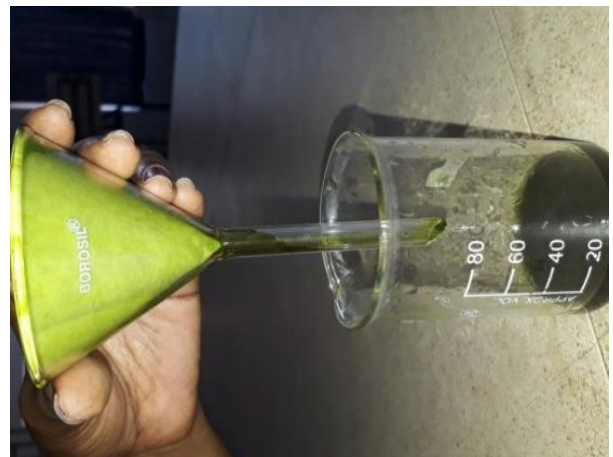

Fig. 8. Filtration

\section{RESULTS AND DISCUSSION}

Neem extract has been prepared by the above mentioned procedure, the neem extract were prepared, now this work was extended by the addition of neem extract with the collagen extracted from the fish scales (Figure 9).

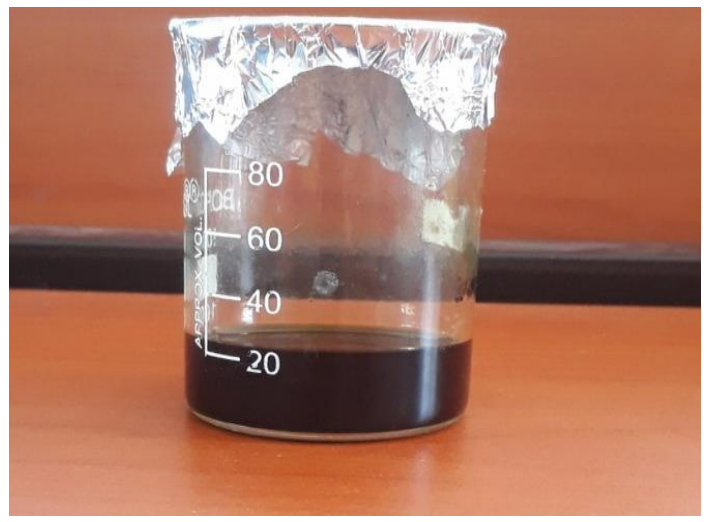

Fig. 9. Collagen

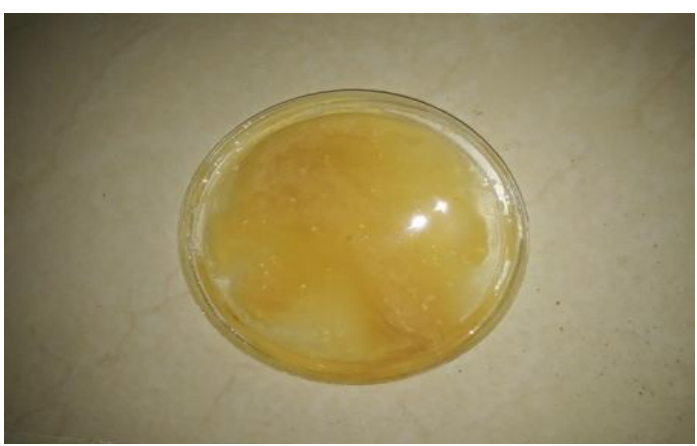

Fig.10. Neem Extract

Obtained neem extract has been shown in Figure 10. The collagen is extracted from the fish scales by the above mentioned methods. Now this collagen is treated with acidic solution and freeze at $-80^{\circ} \mathrm{c}$ and then freeze dried the collagen which produces the collagen as a spongy structure. This proposed work was conducted to compare the speed of recovery between the collagen sponge and the collagen sponges with the neem extract. This work has been initiated with the hope that addition of neem extract is an innovative work and also they are easily available to everyone.

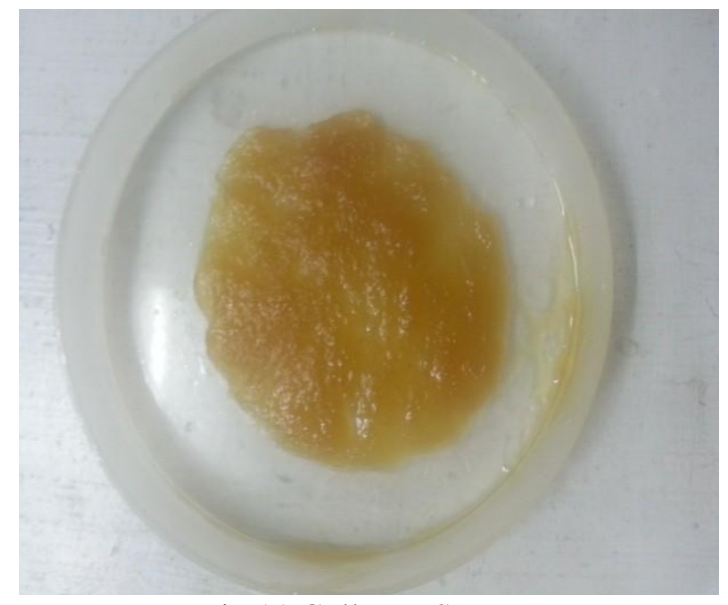

Fig.11.Collagen Sponge

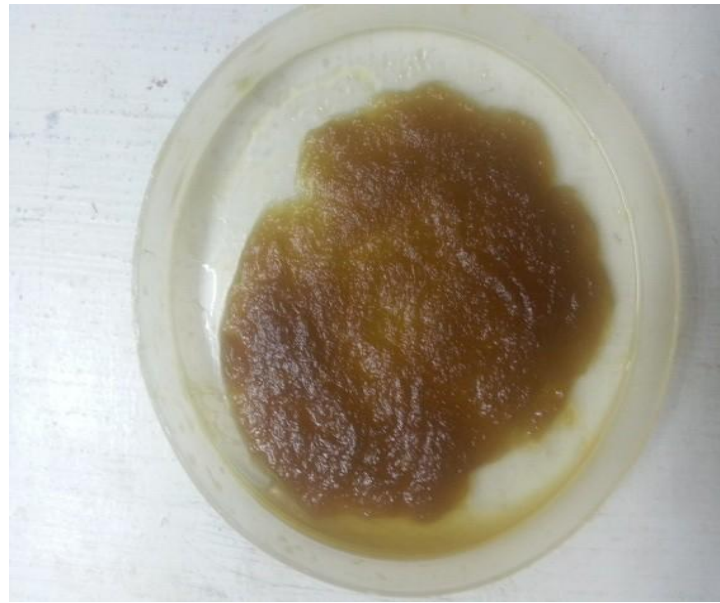

Fig.12.Collagen Sponge with Neem Extract

Prepared collagen sponge is depicted in Figure 11 and Figure 12 shows obtained collagen sponge with neem extract. As per the survey analysis taken from the doctors, patients and pharmacist who resident in Madurai, changing wound dressings regularly or periodically may cause pain, irritation or any sort of discomfort will be felt by patients. They needs around 30-45 minutes for a simple wound dressing in most of the cases. Particularly patients feel difficulty when they are suffered from large wounds such as extensive burns, foot ulcers, pressure ulcers etc. To overcome this problems, the proposed work was proceeded successfully by prepared the collagen sponges with the addition of neem extract which increases the tissue in growth, speed of recovery, cell migration and the anti-bacterial efficacy. Also, this sponge will be cost effective.

\section{CONCLUSION}

Proposed work has been carried out to prepare collagen sponges for wound healing. In order to increase the antimicrobial efficacy ne em extract has been prepared separately and added with the collagen sponges. It would be highly effective and useful for the patients who suffer from chronic wounds for longer duration. Proposed work "Col lagen Based Sponges for Wound Healing" may be extended by takin $g$ the biocompatibility test, due to their direct contact with our tissues. 
Also this work has been planned to extend furthermore by means of comparing the healing duration of collagen sponges and the colla gen sponges prepared with the neem extract. Generally collagen degrades at room temperature hence the prepared collagen can be mixed with cyclodextrins, which increase the stability of collagen.

\section{REFERENCES}

1. GAravinthan, Adithan, et al. "Collagen-based sponge hastens wound healing via decrease of inflammatory cytokines." 3 Biotech 8.12 (2018): 487.

2. Shazeeb, Mohammed Salman, et al. "Developing quantitative MRI parameters to characterize host response and tissue ingrowth into collagen scaffolds." NMR in Biomedicine (2019): e4059.

3. Zhang, Lin, et al. "Immune response of bovine sourced cross-linked collagen sponge for hemostasis." Journal of biomaterials applications 32.7 (2018): 920-931.

4. Zhou, Tian, et al. "Electrospun tilapia collagen nanofibers accelerating wound healing via inducing keratinocytes proliferation and differentiation." Colloids and Surfaces B: Biointerfaces 143 (2016): 415-422.

5. Yan, Ruijian, et al. "A collagen-coated sponge silk scaffold for functional meniscus regeneration." Journal of tissue engineering and regenerative medicine (2018).

6. Sun, Leilei, et al. "Characterization of Pacific cod (Gadus macrocephalus) skin collagen and fabrication of collagen sponge as a good biocompatible biomedical material." Process Biochemistry 63 (2017): 229-235.

7. Sun, Leilei, et al. "Effects of cross-linking on mechanical, biological properties and biodegradation behaviour of Nile tilapia skin collagen sponge as a biomedical material." Journal of the mechanical behaviour of biomedical materials 80 (2018): 51-58.

8. Muthukumar, Thangavelu, et al. "Fish scale collagen sponge incorporated with Macrotyloma uniflorum plant extract as a possible wound/burn dressing material." Colloids and Surfaces B: Biointerfaces 113 (2014): 207-212.

9. Song, Jeong Eun, et al. "Inflammatory response study of gellan gum impregnated duck's feet derived collagen sponges." Journal of Biomaterials science, Polymer edition 27.15 (2016): 1495-1506.

10. Mashiko, Takanobu, et al. "Therapeutic effects of a recombinant human collagen peptide bioscaffold with human adipose-derived stem cells on impaired wound healing after radiotherapy." Journal of tissue engineering and regenerative medicine 12.5 (2018): 1186-1194.

11. Mahmoud, Azza A., and Alaa H. Salama. "Norfloxacin-loaded collagen/chitosan scaffolds for skin reconstruction: Preparation, evaluation and in-vivo wound healing assessment." European Journal of Pharmaceutical Sciences83 (2016): 155-165.

12. Lin, Hsin-Yi, Wen-Chi Tsai, and Shih-Hsing Chang. "Collagen-PVA aligned nanofiber on collagen sponge as bi-layered scaffold for surface cartilage repair." Journal of Biomaterials science, Polymer edition 28.7 (2017): 664-678.

13. Doillon, C. J., et al. "Collagen-based wound dressings: Control of the pore structure and morphology." Journal of biomedical materials research 20.8 (1986): 1219-1228.

14. Fauzi, M. B., et al. "Ovine tendon collagen: Extraction, characterisation and fabrication of thin films for tissue engineering applications." Materials Science and Engineering: C 68 (2016): 163 171.

15. Gould, Lisa J. "Topical collagen-based biomaterials for chronic wounds: rationale and clinical application." Advances in wound care 5.1 (2016): 19-31.

16. M. Anisha, G. Vishnuvarthanan, M. Vallimeenal, L. Pravina and A. Mohammed Ismail, Survey on collagen based sponges for wound healing, Journal of International Pharmaceutical Research 46(1): 802-806, 2019. 